\title{
Inhibition of Adipocyte Differentiation by Anthocyanins Isolated from the Fruit of Vitis coignetiae Pulliat is Associated with the Activation of AMPK Signaling Pathway
}

\author{
Min Ho Han', Hong Jae Kim ${ }^{2,3}$, Jin-Woo Jeong ${ }^{2,3}$, Cheol Park ${ }^{4}$, Byung Woo Kim ${ }^{5}$ and \\ Yung Hyun Choi $\mathbf{i}^{2,3}$ \\ 'Department of Applied Research, National Marine Biodiversity Institute of Korea, Seocheon, Korea \\ ${ }^{2}$ Open Laboratory for Muscular and Skeletal Disease, and Department of Biochemistry, Dongeui University College \\ of Korean Medicine, Busan, Korea \\ ${ }^{3}$ Anti-Aging Research Center, Dongeui University, Busan, Korea \\ ${ }^{4}$ Department of Molecular Biology, College of Natural Sciences, Dongeui University, Busan, Korea \\ ${ }^{5}$ Biopharmaceutical Engineering Major, Division of Applied Bioengineering, College of Engineering, Dongeui \\ University, Busan, Korea
}

\begin{abstract}
Anthocyanins are naturally occurring water-soluble polyphenolic pigments in plants that have been shown to protect against cardiovascular diseases, and certain cancers, as well as other chronic human disorders. However, the anti-obesity effects of anthocyanins are not fully understood. In this study, we investigated the effects of anthocyanins isolated from the fruit of Vitis coignetiae Pulliat on the adipogenesis of 3T3-L1 preadipocytes. Our data indicated that anthocyanins attenuated the terminal differentiation of 3T3-L1 preadipocytes, as confirmed by a decrease in the number of lipid droplets, lipid content, and triglyceride production. During this process, anthocyanins effectively enhanced the activation of the AMP-activated protein kinase (AMPK); however, this phenomenon was inhibited by the co-treatment of compound C, an inhibitor of AMPK. Anthocyanins also inhibited the expression of adipogenic transcription factors, including peroxisome proliferator-activated receptor- $\gamma$, CCAAT/ enhancer-binding protein a and $\mathrm{b}$, and sterol regulatory element-binding protein-1c. In addition, anthocyanins were found to potently inhibit the expression of adipocyte-specific genes, including adipocyte fatty acid-binding protein, leptin, and fatty acid synthase. These results indicate that anthocyanins have potent anti-obesity effects due to the inhibition of adipocyte differentiation and adipogenesis, and thus may have applications as a potential source for an anti-obesity functional food agent.
\end{abstract}

Key words: Vitis coignetiae pulliat, Anthocyanins, 3T3-L1 preadipocytes, Adipogenesis, AMPK

\section{INTRODUCTION}

Obesity is characterized by the overgrowth of adipocytes, which is caused by an increase in the number of adi-

Correspondence to: Yung Hyun Choi, Department of Biochemistry, Dongeui University College of Korean Medicine, 52-57, Yangjeong-ro, Busanjin-gu, Busan 47227, Korea

E-mail: choiyh@deu.ac.kr

This is an Open-Access article distributed under the terms of the Creative Commons Attribution Non-Commercial License (http:// creativecommons.org/licenses/by-nc/3.0) which permits unrestricted non-commercial use, distribution, and reproduction in any medium, provided the original work is properly cited. pocytes due to the division and differentiation of preadipocytes. Adipocytes also store an excess of energy in the form of triglycerides (TGs), and play an important role in releasing energy in the form of glycerol and fatty acids $(1,2)$. In particular, preadipocytes differentiate into mature adipocytes due to high caloric intake, and various stimulatory factors, such as insulin and glucocorticoids $(3,4)$. The differentiation of preadipocytes into mature adipocytes is accompanied by the sequential expression and activation of adipogenic transcription factors, such as peroxisome proliferator-activated receptor- $\gamma(\mathrm{PPAR} \gamma)$, CCAAT/enhancerbinding proteins (C/EBPs), and sterol regulatory elementbinding protein-1c (SREBP-1c), in a coordinated manner 
$(4,5)$. In the final stage of differentiation, adipocytes secrete adipose tissue-specific products, including adipocyte-specific fatty acid binding protein (aP2), and leptin (6-9). After differentiation, lipolytic enzymes, such as fatty acid synthase (Fas), acetyl-CoA carboxylase (ACC), and hormone sensitive lipase, are synthesized to regulate lipid metabolism $(10,11)$.

AMP-activated protein kinase (AMPK), which is recognized as a major regulator of fat metabolism, is a key sensory enzyme controlling energy metabolism $(12,13)$. When AMPK is activated by phosphorylation by the upstream AMPK kinase, it functions as a cellular energy sensor through the regulation of fatty acid and glucose homeostasis $(14,15)$. AMPK also induces inactivation by phosphorylating ACC, an important enzyme for fatty acid oxidation and biosynthesis $(11,16)$. Furthermore, when AMKP is activated, it may protect preadipocyte differentiation with the inhibition of transcription factors. In particular, low levels of AMPK activation are known to be associated with the onset of obesity and diabetes $(17,18)$. Therefore, the discovery of substances that inhibit the differentiation of preadipocytes into adipocytes associated with activation of AMPK signaling would be useful in the treatment of metabolic diseases associated with obesity $(12,18,19)$.

Plant-based materials have been used for thousands of years as traditional medicines in many cultures and are well-known sources for developing anti-obesity compounds. In this study, the fruit of Vitis coignetiae Pulliat (known as meoru in Korea), which has been used in traditional medicine to treat several diseases, was selected from among traditional Korean medicine resources for the development of new anti-obesity active agents. The fruit of $V$. coignetiae contain abundant anthocyanins belonging to the class of flavonoids (20-22). Moreover, several previous studies have shown that the extracts or compounds of the fruit have many pharmacological activities, including hepatoprotective (23), anti-inflammatory (24), vasorelaxant (25), antioxidant $(24,26,27)$, anti-cariogenic (28), chemopreventive (29), and anti-cancer (30-32) properties. Although resveratrol extracted from this fruit has been reported to reduce food intake (33), to our knowledge, the efficacy of anthocyanins isolated from the fruit of $V$. coignetiae as anti-obesity agents and the underlying molecular mechanisms are still unclear. Therefore, we designed this study to evaluate the anti-obesity effect of anthocyanins using 3T3-L1 preadipocytes, and attempted to study the mechanism associated with the inhibition of the adipogenesis of 3T3-L1 cells.

\section{MATERIALS AND METHODS}

Reagents and antibodies. Anthocyanins isolated from the fruit of $V$. coignetiae Pulliat were a generous gift from Dr S.C. Shin (Department of Chemistry, Gyeongsang National University, Jinju, Korea) (22), and $100 \mathrm{mg} /$ $\mathrm{mL}$ concentration stock solution was made by dissolving in distilled water. Dulbecco's modified Eagle's medium (DMEM), bovine calf serum (BCS), and other tissue culture reagents were obtained from WelGENE Inc (Daegu, Korea). 3-(4,5-dimethylthiazol-2-yl)-2,5-diphenyltetrazolium bromide (MTT), differentiation medium (MDI), and TG assay kit were purchased from SigmaAldrich Chemical Co (St. Louis, MO, USA). Bio-Rad protein assay kit was obtained from Bio-Rad Laboratory (Hercules, CA, USA). Primary antibodies (Table 1) were purchased from Santa Cruz Biotechnology, Inc. (Dallas, TX, USA) and Cell Signaling Technology, Inc (Danvers, MA, USA). Horseradish peroxidase (HRP)-conjugated secondary antibodies and enhanced chemiluminescence (ECL) kit were purchased from Amersham Biosciences (Westborough, MA, USA). All other chemicals not specifically mentioned here were purchased from the SigmaAldrich Chemical Co.

Cell culture. The murine 3T3-L1 preadipocytes were purchased from the American Type Culture Collection

Table 1. Primary antibodies used in the present study

\begin{tabular}{llll}
\hline \hline Antibody & Dilution & Product no. & Species of origin and supplier \\
\hline AMPK & $1: 1000$ & sc-74461 & Mouse monoclonal, Santa Cruz Biotechnology, Inc. \\
p-AMPK & $1: 1000$ & 2535 & Rabbit polyclonal, Cell Signaling Technology, Inc. \\
ACC & $1: 1000$ & sc-137104 & Mouse monoclonal, Santa Cruz Biotechnology, Inc. \\
p-ACC & $1: 1000$ & 3661 & Rabbit polyclonal, Cell Signaling Technology, Inc. \\
PPAR $\gamma$ & $1: 1000$ & sc-7273 & Mouse monoclonal, Santa Cruz Biotechnology, Inc. \\
C/EBP $\alpha$ & $1: 1000$ & 2295 & Rabbit polyclonal, Cell Signaling Technology, Inc. \\
C/EBP $\beta$ & $1: 1000$ & 3087 & Rabbit polyclonal, Cell Signaling Technology, Inc. \\
SREBP-1c & $1: 500$ & sc-365513 & Mouse monoclonal, Santa Cruz Biotechnology, Inc. \\
aP2 & $1: 1000$ & sc-18661 & Goat polyclonal, Santa Cruz Biotechnology, Inc. \\
Leptin & $1: 1000$ & sc-48408 & Mouse monoclonal, Santa Cruz Biotechnology, Inc. \\
FAS & $1: 500$ & 3180 & Rabbit polyclonal, Cell Signaling Technology, Inc. \\
Actin & $1: 1000$ & sc-58673 & Mouse monoclonal, Santa Cruz Biotechnology, Inc. \\
\hline
\end{tabular}


(Manassas, VA, USA), and maintained in DMEM containing $4.5 \mathrm{~g} / \mathrm{L} \mathrm{D}$-glucose supplemented with $10 \% \mathrm{BCS}$ and $1 \%$ penicillin/streptomycin in a humidified atmosphere containing $5 \% \mathrm{CO}_{2}$ and $95 \%$ air at $37^{\circ} \mathrm{C}$.

Differentiation of 3T3-L1 preadipocytes. To differentiate the 3T3-L1 preadipocytes into adipocytes, 3T3-L1 preadipocytes were grown in culture plates for 2 day to full confluency. Thereafter, the cells were cultured in a MDI containing $5 \mu \mathrm{g} / \mathrm{mL}$ insulin, $1 \mathrm{mM}$ dexamethasone, and $0.5 \mathrm{mM}$ isobutylmethylxanthine. After 2 day, the medium was changed to DMEM, containing $10 \%$ BCS and $5 \mu \mathrm{g} / \mathrm{mL}$ insulin for 2 day, and subsequently cultured in a normal medium for 4 day. Various concentrations of anthocyanins were added along with the MDI, and 3T3L1 preadipocytes that induced 8-day differentiation were used for various experimental analyses.

MTT assay. To evaluate the cytotoxic ability of anthocyanins, 3T3-L1 preadipocytes were seeded in 6-well plates at a density of $1 \times 10^{4}$ cells per well. After $24 \mathrm{hr}$, the cells were treated with different concentrations of anthocyanins for $72 \mathrm{hr}$. Thereafter, a MTT solution was added to each well at a concentration of $0.5 \mathrm{mg} / \mathrm{mL}$, followed by incubation at $37^{\circ} \mathrm{C}$ in a dark environment for $3 \mathrm{hr}$. The MTT solution was removed, and dimethylsulfoxide (DMSO, $200 \mu \mathrm{L}$ ) was added to dissolve the formazan complex. The viable cells were detected by reading the absorbance of formazan at $540 \mathrm{~nm}$ using an enzyme-linked immunosorbent assay (ELISA) microplate reader (Dynatech Laboratories, Chantilly, VA, USA). The optical density of the formazan formed in the control (untreated) cells was used to represent $100 \%$ viability.

Oil Red $O$ staining. The cells were twice washed gently with ice-cold phosphate-buffered saline (PBS), and fixed with $3.7 \%$ formalin at room temperature for $1 \mathrm{hr}$. Thereafter, the cells were stained with Oil Red O solution $(0.5 \%$ in propylene glycol) for $20 \mathrm{~min}$, and rinsed with $70 \%$ ethanol and PBS. The stained lipid droplets in the cells were then observed under an inverted microscope (Carl Zeiss, Oberkochen, Germany).

Measurement of the intracellular TG content. The cellular TG contents were measured using a commercial TG assay kit according to the manufacturer's instructions. Briefly, the harvested adipocytes were washed twice with ice-cold PBS, and lysed in RIPA lysis buffer. Thereafter, the cells were centrifuged at $13,000 \mathrm{~g}$ for $20 \mathrm{~min}$ at $4^{\circ} \mathrm{C}$, and the supernatants were used to measure the intracellular TG content at $550 \mathrm{~nm}$ using an ELISA.

Protein isolation and Western blot analysis. The cells were harvested, lysed, and the protein concentrations were quantified using a Bio-Rad protein assay, as described in a previous study (34). For Western blotting, equal amounts of protein samples were electrophoretically transferred onto polyvinylidene difluoride membranes (Schleicher \& Schuell, Keene, NH, USA), following electrophoretic separation on sodium-dodecyl sulfate (SDS) gel. Subsequently, the membranes were blocked with 5\% nonfat dry milk/Tris-buffered saline containing $0.1 \%$ Triton X-100 (TBST) for $1 \mathrm{hr}$, and probed overnight with specific primary antibodies at $4^{\circ} \mathrm{C}$. After washing the primary antibodies with TBST, the membranes were incubated with the appropriate HRP-conjugated secondary antibodies for $2 \mathrm{hr}$ at room temperature. The protein bands were detected using an ECL kit, as per the manufacturer's instructions.

Statistical analysis. All numerical data are expressed using mean \pm standard deviation (SD) values. The significance of the differences between the mean values was analyzed using the Student's $t$-test. All statistical analyses were conducted using the Statistical Package for the Social Sciences version 17.0 software (SPSS Inc., Chicago, IL, USA). Values of $p<0.05$ were considered statistically significant.

\section{RESULTS}

Effects of anthocyanins on 3T3-L1 preadipocyte viability. To determine the cytotoxicity of anthocyanins, the 3T3L1 cells were treated with various concentrations of anthocyanins, and the cell viability was assessed using an MTT assay. Our data indicate that anthocyanins exhibited no cytotoxic effect at concentrations $\leq 300 \mu \mathrm{g} /$ $\mathrm{mL}$ (Fig. 1). Therefore, the maximum concentration of anthocyanins was set at $200 \mu \mathrm{g} / \mathrm{mL}$ for future research.

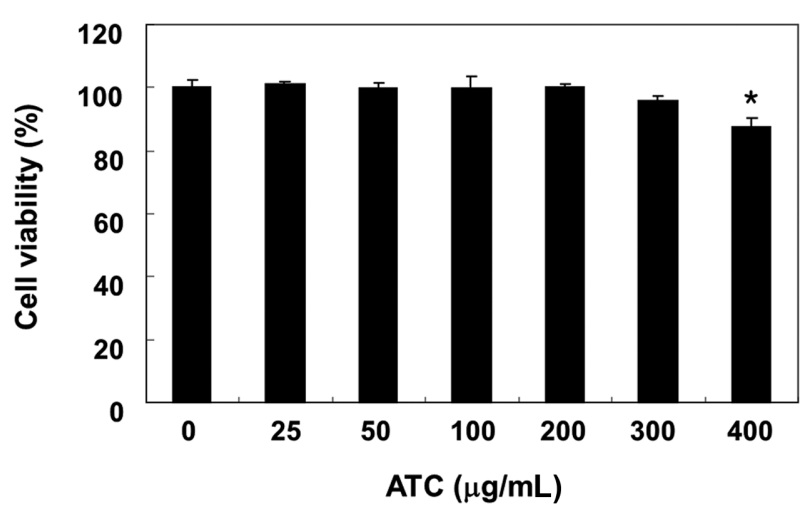

Fig. 1. Effects of anthocyanins on the proliferation of 3T3-L1 mouse preadipocytes. 3T3-L1 cells were treated with various concentrations of anthocyanins (ATC) for $72 \mathrm{hr}$. Cell viability was determined using an MTT assay. The data were expressed as the mean \pm SD of three independent experiments $\left({ }^{*} p<\right.$ 0.05 , vs. untreated control). 
Inhibition of 3T3-L1 preadipocyte differentiation by anthocyanins. To measure the effects of anthocyanins on adipogenesis, 3T3-L1 preadipocytes were differentiated in the presence or absence of anthocyanins. As shown in Fig. 2A and 2B, anthocyanins reduced lipid accumulation in 3T3-L1 adipocytes, as evidenced by the decrease in the cell size and number of lipid droplets in the mature adipocytes in a concentration-dependent manner. Further, it is noteworthy that after treatment with $200 \mu \mathrm{g} / \mathrm{mL}$ anthocyanins, there was about $60 \%$ lipid reduction (Fig. $2 \mathrm{C}$ ), suggesting that anthocyanins could exert an anti-adipogenic effect, through inhibition or delay in the differentiation and adipogenesis of the 3T3-L1 preadipocytes.

Phosphorylation of AMPK and ACC by anthocyanins 3T3-L1 preadipocytes. Because the activation of AMKP inhibits preadipocyte differentiation and adipogenesis through increased phosphorylation of ACC, a substrate of
AMPK $(11,15)$, we analyzed the levels of phosphorylated AMPK, to investigate whether AMPK is activated by anthocyanins during 3T3-L1 differentiation. Our results indicated that anthocyanins remarkably enhanced the phosphorylation of AMPK and ACC without altering the expression of their total protein expression, indicating the activation of the AMPK pathway (Fig. 3). However, when compound $\mathrm{C}$, an AMPK specific inhibitor, was pretreated in the 3T3-L1 cells, the phosphorylation of both AMPK and ACC induced by anthocyanins was significantly reduced (Fig. 4).

Effects of anthocyanins on the expression of adipogenic transcription factors. The differentiation of preadipocytes into adipocytes is coordinated by a complex process that involves the sequential activation of multiple adipogenic transcription factors $(4,5)$. To investigate the role of anthocyanins in reducing fat accumulation during

ATC $(\mu \mathrm{g} / \mathrm{mL})$

\begin{tabular}{ccccc}
\hline 0 & 0 & 50 & 100 & 200 \\
- & + & + & + & +
\end{tabular}

(A)
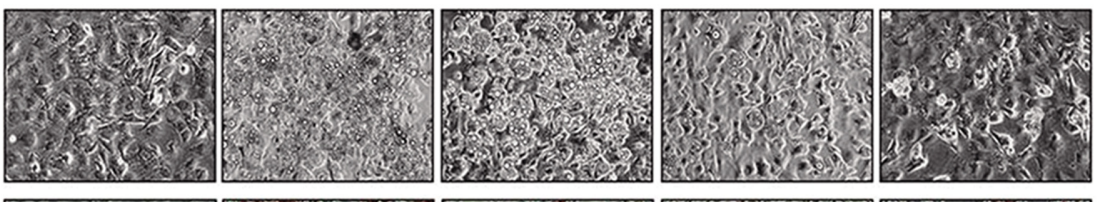

(B)

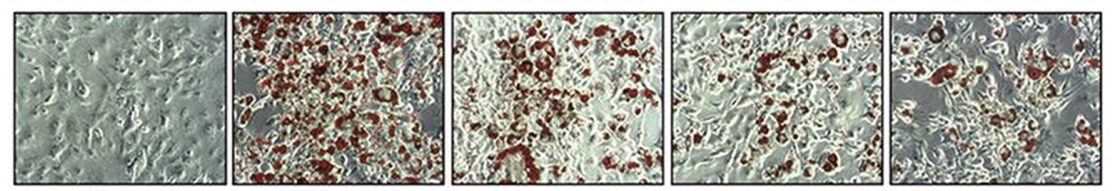

(C)

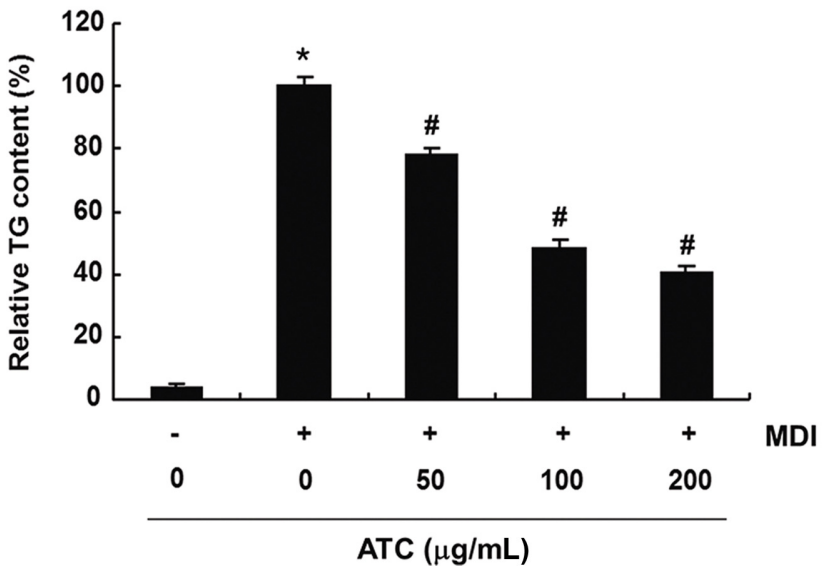

Fig. 2. Effects of anthocyanins on intracellular lipid accumulation and TG content in the differentiated 3T3-L1 cells. 3T3-L1 preadipocytes were treated with the indicated concentrations of anthocyanins during differentiation. (A) and (B) on day 8 , (A) the cells were directly observed via an inverted microscope, or (B) the cells were fixed and stained with Oil Red $O$ to visualize lipid droplets (Magnification, $\times 200$ ). (C) The TG content was measured to quantify the intracellular lipid content at $500 \mathrm{~nm}$ wavelength, using the ELISA reader. The data were expressed as the mean \pm SD of three independent experiments $\left({ }^{*} p<0.05\right.$, vs. undifferentiated control; $" p<0.05$, vs. differentiated control). 


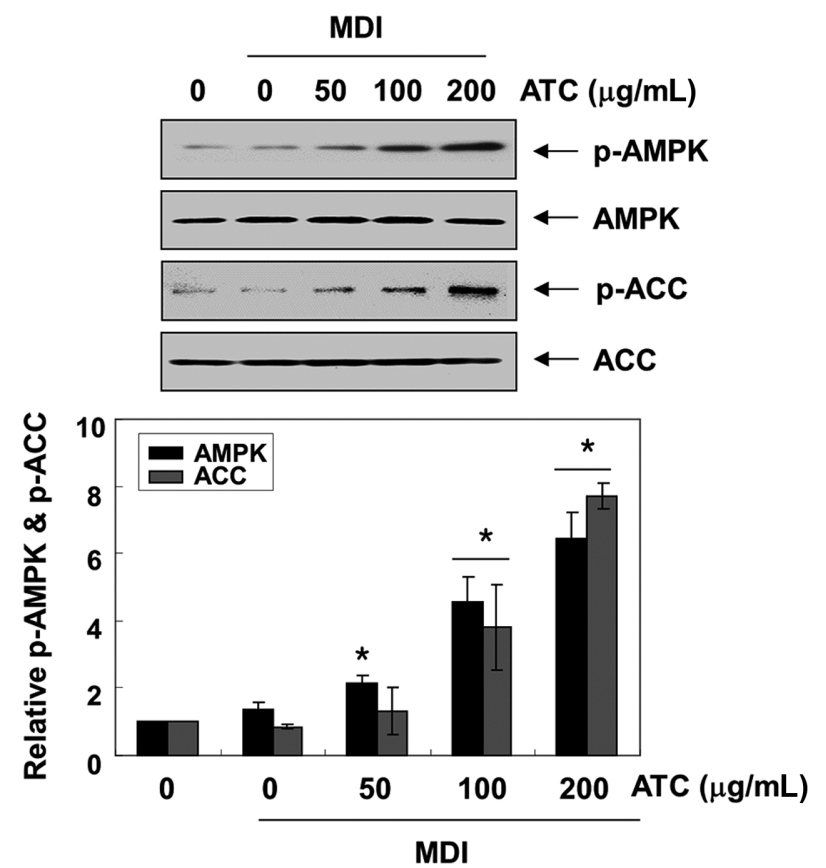

Fig. 3. Activation of AMPK signaling by anthocyanins in the differentiated 3T3-L1 cells. The proteins were isolated from cells grown under the same conditions (as shown in Fig. 2), and the cellular proteins were separated electrophoretically using SDS-polyacrylamide gels, and transferred onto membranes. The total protein levels of AMPK and ACC, and their phosphorylated states ( $\mathrm{p}-\mathrm{AMPK}$ and $\mathrm{p}-\mathrm{ACC}$, respectively) were determined using the indicated antibodies and an ECL detection system. Actin was used as an internal control. The relative ratios of the phosphorylation types relative to the total protein amounts in the results of the Western blotting are presented at the bottom. The data were expressed as the mean \pm SD of three independent experiments $\left({ }^{*} p<0.05\right.$, vs. undifferentiated control).

the adipogenesis of 3T3-L1 preadipocytes, we examined the effects of anthocyanins on the expression of these adipogenic transcription factors, such as PPAR $\gamma, \mathrm{C} / \mathrm{EBP} \alpha / \beta$, and SREBP-1c. The immunoblotting results indicated that following anthocyanins treatment, the increased expression of the transcription factors was largely inhibited in a concentration-dependent manner (Fig. 5). These results suggest that the inhibition of lipid accumulation by anthocyanins is associated with the suppression of the expression of adipogenic transcription factors.

Effects of anthocyanins on the expression of adipocyte-related genes. We further examined the influence of anthocyanins on the expression of adipocyte-related markers, such as aP2, leptin, and FAS (7,9). Fig. 6 shows that after anthocyanins administration, the enhanced expression of aP2, leptin, and FAS protein in differentiated 3T3L1 cells decreased in a concentration-dependent manner,

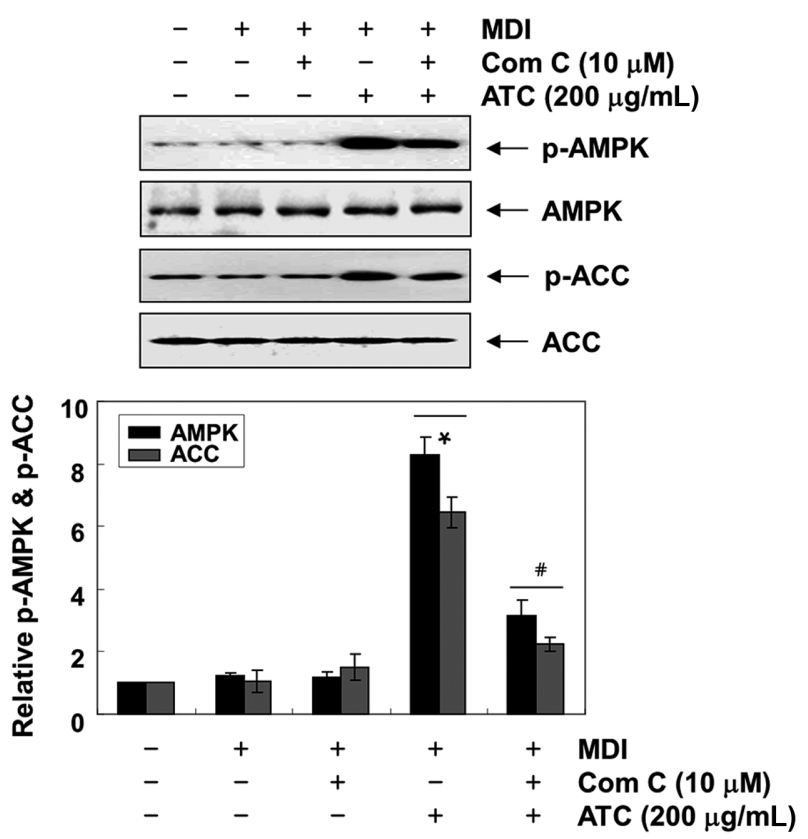

Fig. 4. Effects of AMPK inhibitor on the anthocyanins-induced activation of AMPK in the differentiated 3T3-L1 cells. 3T3-L1 cells were pre-treated with compound C, an AMPK inhibitor, for $1 \mathrm{hr}$, and then treated with $200 \mu \mathrm{g} / \mathrm{mL}$ anthocyanins. On day 8 , completely differentiated cells were lysed, and the cellular proteins were separated electrophoretically using SDS-polyacrylamide gels, and transferred onto membranes. The membranes were probed with the indicated antibodies. Proteins were observed using an ECL detection system. Actin was used as an internal control. The relative ratios of the phosphorylation types relative to the total protein amounts in the results of the Western blotting are presented at the bottom. The data were expressed as the mean $\pm S D$ of three independent experiments $\left({ }^{*} p<0.05\right.$, vs. undifferentiated control; ${ }^{\#} p<0.05$, vs. differentiated cells treated with anthocyanins alone).

compared to that in the control group. These results support the hypothesis that the anti-adipogenic effect of anthocyanins is associated with the downregulation of the expression of adipocyte-related markers involved in lipid metabolism.

\section{DISCUSSION}

According to the present results of this study, the lipid accumulation and TG content in the cytoplasm of differentiated 3T3-L1 cells was actively increased, and was significantly inhibited by anthocyanins treatment in a concentration-dependent manner. Adipocyte differentiation is accompanied by lipid and TG accumulation; therefore, the results indicate that anthocyanins treatment significantly suppressed the differentiation of 3T3-L1 preadipocytes.

It has also been reported that the AMPK signaling pathway is a target for the energy balance and metabolic disor- 
(A)

$$
\begin{array}{llllll}
0 & 0 & 50 & 100 & 200 & \text { ATC }(\mu \mathrm{g} / \mathrm{mL})
\end{array}
$$

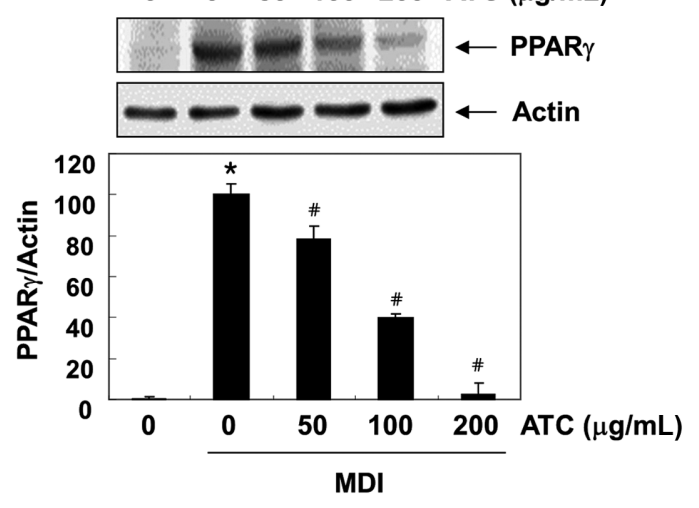

(C)

$$
\begin{array}{llllll}
0 & \multicolumn{4}{c}{\text { MDI }} & \\
\cline { 3 - 4 } & 0 & 50 & 100 & 200 & \text { ATC }(\mu \mathrm{g} / \mathrm{mL})
\end{array}
$$

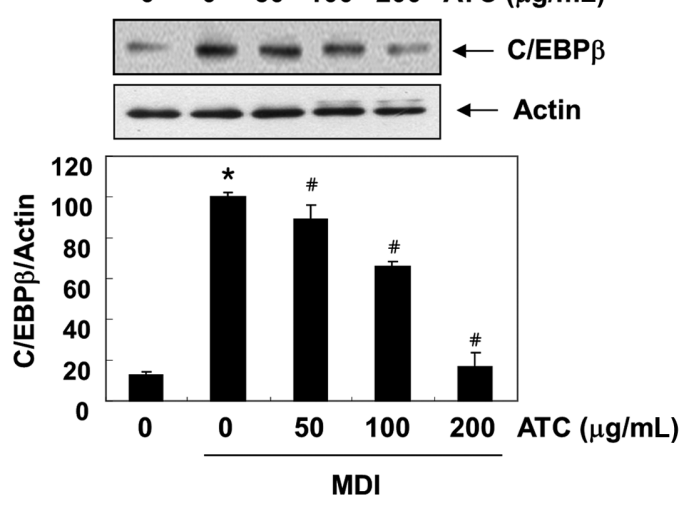

(B)

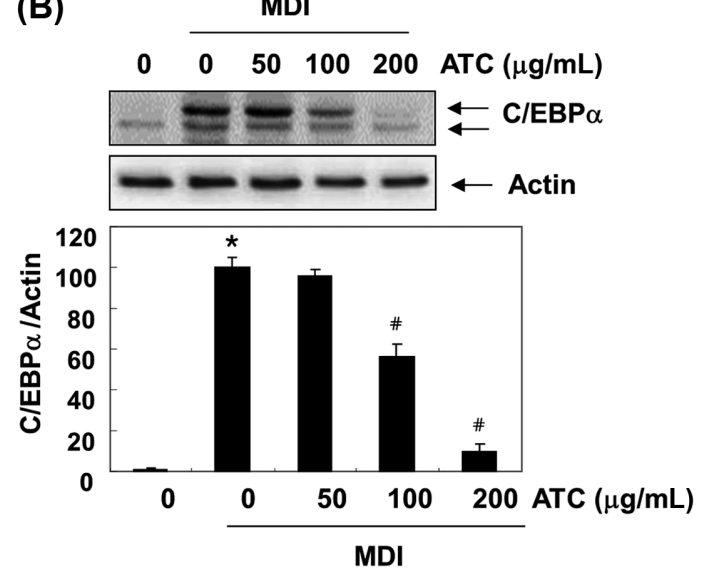

(D)

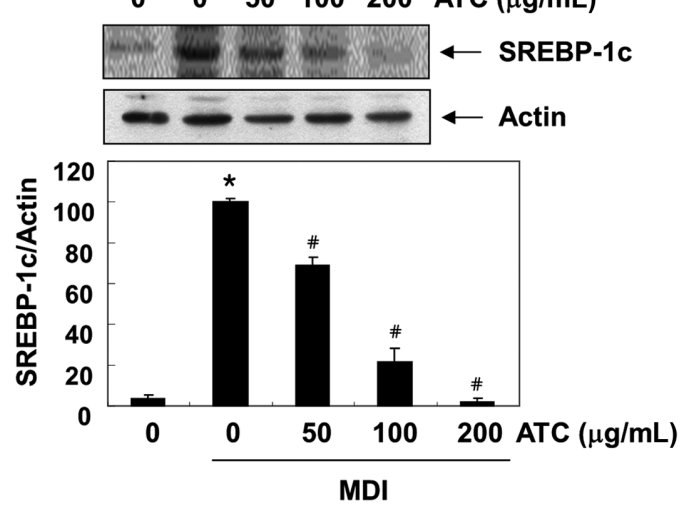

Fig. 5. Effects of anthocyanins on the levels of adipogenic transcription factors expression in the differentiated 3T3-L1 cells. At day 0 , confluent 3T3-L1 preadipocytes were treated with various concentrations of anthocyanins in the absence or presence of an MDI differentiation medium for $48 \mathrm{hr}$. On day 8, completely differentiated cells were lysed to extract the total protein. The cellular proteins were separated electrophoretically using SDS-polyacrylamide gels, and transferred onto membranes. The membranes were probed with the indicated antibodies. The proteins were visualized using an ECL detection system. Actin was used as an internal control. The relative ratios of the expression in the results of Western blotting are presented at the bottom of each result as the relative values of the expressions in the MDI-treated group. The data were expressed as the mean \pm SD of three independent experiments ( ${ }^{*} p<0.05$, vs. undifferentiated control; ${ }^{*} p<0.05$, vs. differentiated control).

ders involved in the maintenance of lipid and cholesterol homeostasis. Accumulating evidence suggests that AMPK is inactivated by lower phosphorylation levels during preadipocyte differentiation and adipogenesis $(15,35)$. In addition, AMPK activation by phosphorylation can reduce the degree of obesity by inhibiting the differentiation of adipocytes, by altering the expression and activity of enzymes and proteins involved in lipid metabolism $(14,35)$. Furthermore, ACC, a downstream substrate of AMPK, is a rate-limiting enzyme that limits the critical rate of fatty acid synthesis and oxidation, and inhibits the onset and progression of obesity, by reducing fatty acid and lipid synthesis $(11,36)$. Therefore, AMPK signaling has attracted researchers' attention as a molecular target to combat obesity. To determine whether AMPK activation is involved in anthocyanins-mediated attenuation of adipocyte differ- entiation and adipogenesis, we determined whether anthocyanins can activate AMPK. According to our results, compound $\mathrm{C}$ effectively suppressed the phosphorylation levels of AMPK and ACC induced by anthocyanins, indicating that the activation of AMPK is involved in the inhibition of adipocyte differentiation by anthocyanins.

Accumulating evidence suggests that the differentiation of preadipocytes into adipocytes is regulated by a complicated process that is continuously regulated by various transcription factors. Among them, C/EBP $\beta$ is induced in the early stage of differentiation, and is known to initiate the expression of PPAR $\gamma$ and $\mathrm{C} / \mathrm{EBP} \alpha$, which are major regulators of adipogenesis and adipogenesis in late differentiation $(4,5)$. In addition, SREBP-1c is involved in lipid metabolism, and is an additional regulator of FAS. SREBP1c also enhances the expression of downstream genes 
(A)

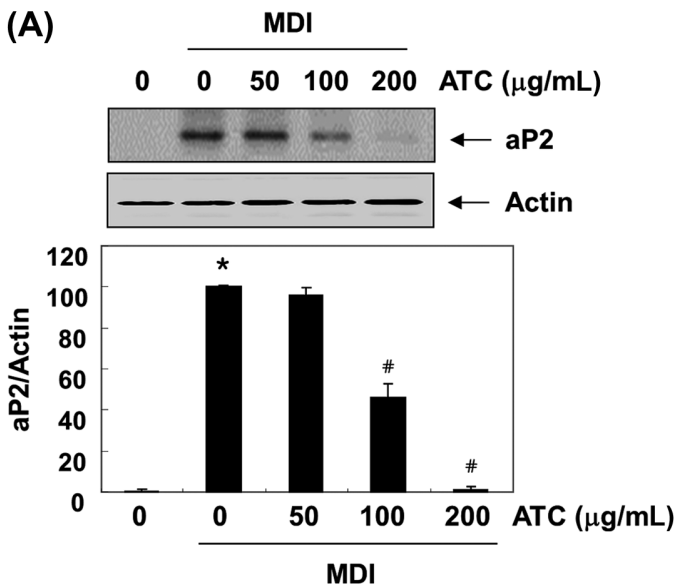

(B)

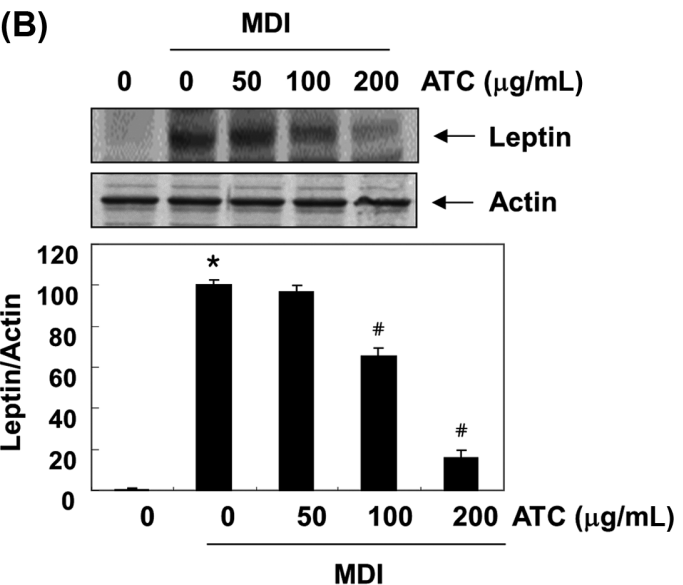

(C) MDI

$0 \quad 0 \quad 50100200$ Anthocyanins $(\mu \mathrm{g} / \mathrm{mL})$

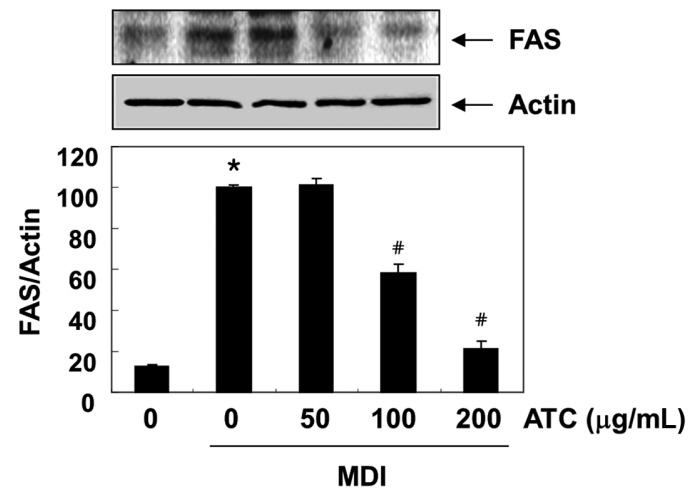

Fig. 6. Effects of anthocyanins on the levels of adipocyte expressed genes expression in the differentiated 3T3-L1 cells. The proteins were isolated cells grown under the same conditions (as shown in Fig. 2), and the cellular proteins were separated electrophoretically using SDS-polyacrylamide gels, and transferred onto membranes. The membranes were probed with the indicated antibodies. The proteins were visualized using an ECL detection system. Actin was used as an internal control. The relative ratios of the expression in the results of Western blotting are presented at the bottom of each result as the relative values of the expressions in the MDI-treated group. The data were expressed as the mean \pm SD of three independent experiments $\left({ }^{*} p<0.05\right.$, vs. undifferentiated control; ${ }^{*} p<0.05$, vs. differentiated control).

associated with preadipocyte differentiation and fatty acid metabolism $(37,38)$. In addition to activating adipogenic transcription factors, transactivating adipocyte-specific genes, such as aP2, leptin, and FAS, are also very important for the differentiation of preadipocytes into mature adipocytes $(8,17)$. Thus, the inhibition of adipogenic transcription and adipocyte-specific factors will reduce the adipocyte differentiation associated with fatty acid and TG biosynthesis. To investigate the role of anthocyanins in regulating expression levels of adipogenic transcription factors in the adipogenic process of 3T3-L1 cells, we compared their expression levels in the presence and absence of anthocyanins. Our data showed that anthocyanin treatment of 3T3-L1 adipocytes significantly reduced the expression of the four transcription factors tested during adipocyte differentiation. These results showed that anthocyanins blocked the differentiation of adipocytes by sup- pressing the expression of transcription factors involved in different differentiation stages. In addition, anthocyanins also reduced the expression of adipocyte markers on 3T3L1 cells, suggesting that anthocyanins strongly suppress the synthesis of TGs and adipocyte differentiation.

In summary, the present results indicate that anthocyanins prevented adipocyte differentiation and adipogenesis in 3T3-L1 cells by reducing adipogenic transcription factors, without cytotoxicity and their downstream target genes. In this process, anthocyanins also activated the AMPK, and activation of the AMPK pathway by anthocyanins may be a potential strategy to prevent obesity.

\section{ACKNOWLEDGMENTS}

This work was supported by Blue-Bio Industry Regional Innovation Center (RIC08-06-07) at Dongeui University 
as a RIC program under Ministry of Trade, Industry \& Energy and Busan city and Basic Science Research Program through the National Research Foundation of Korea (NRF) grant funded by the Korea government (2015R1A2A2A01004633).

Received September 10, 2017; Revised November 23, 2017; Accepted December 7, 2017

\section{REFERENCES}

1. Frühbeck, G., Méndez-Giménez, L., Fernández-Formoso, J.A., Fernández, S. and Rodríguez, A. (2014) Regulation of adipocyte lipolysis. Nutr. Res. Rev., 27, 63-93.

2. Berk, P.D. and Verna, E.C. (2016) Nonalcoholic fatty liver disease: lipids and insulin resistance. Clin. Liver Dis., 20, 245-262.

3. Ali, A.T., Hochfeld, W.E., Myburgh, R. and Pepper, M.S. (2013) Adipocyte and adipogenesis. Eur. J. Cell Biol., 92, 229-236.

4. Mota de Sá, P., Richard, A.J., Hang, H. and Stephens, J.M. (2017) Transcriptional regulation of adipogenesis. Compr. Physiol., 7, 635-674.

5. Guo, L., Li, X. and Tang, Q.Q. (2015) Transcriptional regulation of adipocyte differentiation: a central role for CCAAT/ enhancer-binding protein (C/EBP) $\beta$. J. Biol. Chem., 290, 755-761.

6. Kralisch, S. and Fasshauer, M. (2013) Adipocyte fatty acid binding protein: a novel adipokine involved in the pathogenesis of metabolic and vascular disease? Diabetologia, 56, $10-21$.

7. Wang, S., Moustaid-Moussa, N., Chen, L., Mo, H., Shastri, A., Su, R., Bapat, P., Kwun, I. and Shen, C.L. (2014) Novel insights of dietary polyphenols and obesity. J. Nutr. Biochem., 25, 1-18.

8. Stern, J.H., Rutkowski, J.M. and Scherer, P.E. (2016) Adiponectin, leptin, and fatty acids in the maintenance of metabolic homeostasis through adipose tissue crosstalk. Cell Metab., 23, 770-784.

9. Pandit, R., Beerens, S. and Adan, R.A.H. (2017) Role of leptin in energy expenditure: the hypothalamic perspective. Am. J. Physiol. Regul. Integr. Comp. Physiol., 312, R938R947.

10. Nakamura, M.T., Yudell, B.E. and Loor, J.J. (2014) Regulation of energy metabolism by long-chain fatty acids. Prog. Lipid Res., 53, 124-144.

11. Coles, C.A. (2016) Adipokines in healthy skeletal muscle and metabolic disease. Adv. Exp. Med. Biol., 900, 133-160.

12. Jeon, S.M. (2016) Regulation and function of AMPK in physiology and diseases. Exp. Mol. Med., 48, e245.

13. Garcia, D. and Shaw, R.J. (2017) AMPK: mechanisms of cellular energy sensing and restoration of metabolic balance. Mol. Cell, 66, 789-800.

14. Coughlan, K.A., Valentine, R.J., Ruderman, N.B. and Saha, A.K. (2013) Nutrient excess in AMPK downregulation and insulin resistance. J. Endocrinol. Diabetes Obes., 1, 1008.

15. Han, J.Y., Park, S.H., Yang, J.H., Kim, M.G., Cho, S.S., Yoon, G., Cheon, S.H. and Ki, S.H. (2014) Licochalcone suppresses LXR $\alpha$-induced hepatic lipogenic gene expression through AMPK/Sirt1 pathway activation. Toxicol. Res., 30, 19-25.

16. Mount, P., Davies, M., Choy, S.W., Cook, N. and Power, D. (2015) Obesity-related chronic kidney disease-The role of lipid metabolism. Metabolites, 5, 720-732.

17. Kadowaki, T., Yamauchi, T., Waki, H., Iwabu, M., OkadaIwabu, M. and Nakamura, M. (2011) Adiponectin, adiponectin receptors, and epigenetic regulation of adipogenesis. Cold Spring Harb. Symp. Quant. Biol., 76, 257-265.

18. Feng, S., Reuss, L. and Wang, Y. (2016) Potential of natural products in the inhibition of adipogenesis through regulation of PPAR $\gamma$ expression and/or its transcriptional activity. Molecules, 21, E1278.

19. Baek, J.H., Kim, N.J., Song, J.K. and Chun, K.H. (2017) Kahweol inhibits lipid accumulation and induces glucoseuptake through activation of AMP-activated protein kinase (AMPK). BMB Rep., 50, 566-571.

20. Choi, J.Y., Lee, S.J., Lee, S.J., Park, S., Lee, J.H., Shim, J.H., Abd El-Aty, A.M., Jin, J.S., Jeong, E.D., Lee, W.S. and Shin, S.C. (2010) Analysis and tentative structure elucidation of new anthocyanins in fruit peel of Vitis coignetiae Pulliat (meoru) using LC-MS/MS: contribution to the overall antioxidant activity. J. Sep. Sci., 33, 1192-1197.

21. Koyama, K., Kamigakiuchi, H., Iwashita, K., Mochioka, R. and Goto-Yamamoto, N. (2017) Polyphenolic diversity and characterization in the red-purple berries of East Asian wild Vitis species. Phytochemistry, 134, 78-86.

22. Yun, J.W., Lee, W.S., Kim, M.J., Lu, J.N., Kang, M.H., Kim, H.G., Kim, D.C., Choi, E.J., Choi, J.Y., Kim, H.G., Lee, Y.K., Ryu, C.H., Kim, G., Choi, Y.H., Park, O.J. and Shin, S.C. (2010) Characterization of a profile of the anthocyanins isolated from Vitis coignetiae Pulliat and their anti-invasive activity on HT-29 human colon cancer cells. Food Chem. Toxicol., 48, 903-909.

23. Oshima, Y., Namao, K., Kamijou, A., Matsuoka, S., Nakano, M., Terao, K. and Ohizumi, Y. (1995) Powerful hepatoprotective and hepatotoxic plant oligostilbenes, isolated from the Oriental medicinal plant Vitis coignetiae (Vitaceae). Experientia, 51, 63-66.

24. Pak, W., Takayama, F., Hasegawa, A., Mankura, M., Egashira, T., Ueki, K., Nakamoto, K., Kawasaki, H. and Mori, A. (2012) Water extract of Vitis coignetiae Pulliat leaves attenuates oxidative stress and inflammation in progressive NASH rats. Acta. Med. Okayama, 66, 317-327.

25. Ha, S.K., Park, H.Y., Ryu, M.R., Kim, Y. and Park, Y. (2016) Endothelium-dependent vasorelaxant effects of dealcoholized wine powder of wild grape (Vitis coignetiae) in the rat thoracic aorta. Evid. Based Complement. Alternat. Med., 2016, 6846084.

26. Jang, J.H. and Lee, J.S. (2011) Antihypertensive sngiotensin I-converting enzyme inhibitory activity and antioxidant activity of Vitis hybrid-Vitis coignetiae red wine made with Saccharomyces cerevisiae. Mycobiology, 39, 137-139.

27. Weidner, S., Rybarczyk, A., Karamać, M., Król, A., Mostek, A., Grębosz, J. and Amarowicz, R. (2013) Differences in the phenolic composition and antioxidant properties between Vitis coignetiae and Vitis vinifera seeds extracts. Molecules, 18, 3410-3426. 
28. Yano, A., Kikuchi, S., Takahashi, T., Kohama, K. and Yoshida, Y. (2012) Inhibitory effects of the phenolic fraction from the pomace of Vitis coignetiae on biofilm formation by Streptococcus mutans. Arch. Oral Biol., 57, 711-719.

29. Arimoto-Kobayashi, S., Zhang, X., Yuhara, Y., Kamiya, T., Negishi, T. and Okamoto, G. (2013) Chemopreventive effects of the juice of Vitis coignetiae Pulliat on two-stage mouse skin carcinogenesis. Nutr. Cancer, 65, 440-450.

30. Shin, D.Y., Lee, W.S., Kim, S.H., Kim, M.J., Yun, J.W., Lu, J.N., Lee, S.J., Tsoy, I., Kim, H.J., Ryu, C.H., Kim, G.Y., Kang, H.S., Shin, S.C. and Choi, Y.H. (2009) Anti-invasive activity of anthocyanins isolated from Vitis coignetiae in human hepatocarcinoma cells. J. Med. Food, 12, 967-972.

31. Jung, D.B., Lee, H.J., Jeong, S.J., Lee, H.J., Lee, E.O., Kim, Y.C., Ahn, K.S., Chen, C.Y. and Kim, S.H. (2011) Rhapontigenin inhibited hypoxia inducible factor 1 alpha accumulation and angiogenesis in hypoxic PC-3 prostate cancer cells. Biol. Pharm. Bull., 34, 850-855.

32. Lu, J.N., Lee, W.S., Kim, M.J., Yun, J.W., Jung, J.H., Yi, S.M., Jeong, J.H., Kim, H.J., Choi, Y.H., Kim, G.S., Ryu, C.H. and Shin, S.C. (2014) The inhibitory effect of anthocyanins on Akt on invasion and epithelial-mesenchymal transi- tion is not associated with the anti-EGFR effect of the anthocyanins. Int. J. Oncol., 44, 1756-1766.

33. Kim, S.J., Lee, Y.H., Han, M.D., Mar, W., Kim, W.K. and Nam, K.W. (2010) Resveratrol, purified from the stem of Vitis coignetiae Pulliat, inhibits food intake in C57BL/6J mice. Arch. Pharm. Res., 33, 775-780.

34. You, M.K., Kim, H.J., Rhyu, J. and Kim, H.A. (2017) Pear pomace ethanol extract improves insulin resistance through enhancement of insulin signaling pathway without lipid accumulation. Nutr. Res. Pract., 11, 198-205.

35. Angin, Y., Beauloye, C., Horman, S. and Bertrand, L. (2016) Regulation of carbohydrate metabolism, lipid metabolism, and protein metabolism by AMPK. EXS, 107, 23-43.

36. Bijland, S., Mancini, S.J. and Salt, I.P. (2013) Role of AMPactivated protein kinase in adipose tissue metabolism and inflammation. Clin. Sci. (Lond.), 124, 491-507.

37. Xu, X., So, J.S., Park, J.G. and Lee, A.H. (2013) Transcriptional control of hepatic lipid metabolism by SREBP and ChREBP. Semin. Liver Dis., 33, 301-311.

38. Zhou, X., Xu, J., Shi, Y. and Ye, J.M. (2015) Discovery of novel anti-diabetic drugs by targeting lipid metabolism. Curr. Drug Targets, 16, 1372-1380. 